\title{
Electrochemical Immunosensors for the Diagnosis of Celiac Disease
}

\author{
Katharina Anne Scherf*, Peter Koehler, Herbert Wieser \\ Deutsche Forschungsanstalt für Lebensmittelchemie, Leibniz Institut, Freising, Germany \\ Email: "katharina.konitzer@tum.de
}

Received 29 December 2014; accepted 15 January 2015; published 20 January 2015

Copyright (C) 2015 by authors and Scientific Research Publishing Inc.

This work is licensed under the Creative Commons Attribution International License (CC BY). http://creativecommons.org/licenses/by/4.0/

(c) (i) Open Access

\begin{abstract}
Celiac disease is a permanent intolerance to gluten proteins of wheat, rye, barley, and oats in genetically susceptible individuals. The clinical picture is characterized by inflammation and damage of the small intestinal mucosa and malabsorption of essential nutrients. Therapeutically, a lifelong strict gluten-free diet is necessary. The diagnosis of celiac disease is complex and includes symptomatology, serology, small intestinal histology, and genetic status. Serological testing plays a central role within the diagnostic procedure and is based on the measurement of disease-specific antibodies against gluten proteins (antigen) and tissue transglutaminase (autoantigen). Immunofluorescence detection and enzyme-linked immunosorbent assays are currently most often applied for antibody testing. However, these tests are expensive and time-consuming. Therefore, simple and rapid alternative methods have been developed during the last years, and electrochemical immunosensors seem to be the most promising analytical tools. The architecture of these sensors may comprise the following elements: working and reference electrodes, covalent or noncovalent binding of the antigen to the surface of the working electrode by means of a functional monolayer, and blocking of unreacted binding sites. The analytical procedure is initiated by adding the analyte (serum antibodies) and an analyte-specific second antibody, which is usually labeled with an enzyme. The special reaction of the enzyme with an appropriate substrate results in a product that initiates a current that can be measured by different electrical methods. A number of different electrochemical immunosensors variable in different electrodes, binding systems, secondary antibodies, and current measurements have been developed. Most of them have been tested with real human serum samples of celiac patients and healthy individuals, and some of them reached disease sensitivity and specificity comparable with traditional analytical systems. Thus, electrochemical immunosensors can be promising alternatives to existing diagnostic tests in the future. They are simple, reliable, robust, user-friendly, and cost-effective tools with short operation times.
\end{abstract}

*Corresponding author. 
Keywords

Celiac Disease, Diagnosis, Electrochemical Immunosensors, Serum Antibodies

\section{Introduction}

Celiac disease (CD), also known as celiac sprue or gluten-sensitive enteropathy, is a permanent intestinal intolerance to dietary wheat, rye, barley, and oat proteins (called gluten) in genetically susceptible individuals (overview in [1]). The clinical picture of CD is characterized by inflammation and damage of the upper small intestinal mucosa and by extraintestinal symptoms mainly caused by malabsorption of nutrients. Therapeutically, a lifelong strict gluten-free diet (GFD) is necessary. The prevalence of CD was underestimated for a long time. Modern diagnostic methods, however, revealed that CD, with a prevalence of around $1 \%$, is one of the most frequent food intolerances in many parts of the world. Due to variable intestinal and extraintestinal symptoms or even asymptomatic appearance, the diagnosis of CD is complex and requires a high level of clinical suspicion. The precise diagnosis is extremely important for persons suspected to have CD. False-positive results needlessly force individuals to a lifelong GFD, which is a big challenge for the persons affected and their families. Falsenegative results burden potential patients with permanent impairment of health.

Diagnostic approaches have changed during the last decades, owing to a better understanding of the pathomechanism of $C D$ and the availability of specific serological tests. Serological testing plays a central role within the diagnostic procedure and is based on the measurement of CD-specific serum antibodies appearing in the blood in response to gluten intake. Immunofluorescence detection or enzyme-linked immunosorbent assays (ELISA) are currently most often applied for the quantitative determination of CD-specific serum antibodies. However, these tests require qualified personnel and laboratory facilities, and results are available only after a time delay. Therefore, several simple and rapid alternative methods have been developed during the last years. Electrochemical immunosensors (ECISs) seem to be one of the most promising diagnostic tools; an overview on recent developments is provided in the present article.

\section{Diagnosis of Celiac Disease}

The diagnostic scheme of CD consists of the following steps: 1) symptomatology; 2) serology; 3) small intestinal histology; 4) genetic status [1]. Finally, the improvement on a GFD confirms the diagnosis of CD. The primary care doctor has a central role in the process of diagnosis. Well-known symptoms of CD should prompt the physician to initiate serological testing. After positive serologic findings, the histological judgement of biopsies from the small intestinal mucosa is commonly regarded as the gold standard for CD diagnosis. Biopsy specimens of active CD demonstrate a flat mucosa with the absence of intestinal villi, a cellular infiltrate of the lamina propria, and an increase in the numbers of intraepithelial lymphocytes. The intestinal biopsy can be omitted in children and adolescents with high levels of CD-specific serum antibodies exceeding ten times the upper limit of normal [2]. After setting the diagnosis of CD, a strict GFD is essential for CD therapy. For the assessment of adherence to a GFD, serological tests are usually utilized. Altogether, serum antibody tests are important as a noninvasive diagnostic aid in symptomatic CD patients, to select patients for intestinal biopsy, and to control compliance to a GFD.

CD-specific serum antibodies belong to the immunoglobulin (Ig) classes A and G and are directed against gluten proteins (external antigen) and tissue transglutaminase (TG2, autoantigen). The levels of these antibodies in patients' blood are significantly increased in untreated CD and decreased on a GFD. Thus, antibody tests are not only useful for the diagnosis of CD but also for follow-up of adherence to the GFD. Initial serological tests were developed using antibodies against gliadin, the alcohol-soluble fraction of wheat gluten. However, antigliadin antibodies (AGAs) lost diagnostic significance due to their relatively poor sensitivity and specificity. The testing of children may be an exception [3]. AGA tests were first based on immunofluorescent staining of the antigen-antibody complex and then on ELISAs. Currently, IgA and IgG (in the case of IgA deficiency) antibodies against gliadin, endomysium (EMAs), deamidated gliadin peptides (DGPAs) and the autoantigen TG2 (TGAs) are in use. EMA tests are based on indirect immunofluorescence using primate tissues as antigens, and DGPAs and TGAs are based on ELISAs using deamidated gliadin peptides and TG2 as antigens, respectively. A 
combination of different tests is necessary to yield positive and negative predictive values near $100 \%$ [4]. All these tests require qualified personnel and laboratory facilities, and results are available only after a time delay. Therefore, the search for simple and rapid diagnostic methods has been intensified during the last decade. Electrochemical immunosensors have been shown to be most promising, because they are easy to handle, inexpensive, and fast.

\section{Electrochemical Immunosensors}

The fundamental basis of immunosensors is the specificity of the molecular recognition of antigens by antibodies to form a stable complex combined with solid-state devices, in which the immunochemical reaction is coupled to a transducer [5]. Immunosensors can be categorized based on the detection principle applied (e.g., electrochemical, optical, and microgravimetric immunosensors). There are many recent developments in the immunosensor field, for example in environmental science, pharmaceutical and food industries, and in clinical diagnostics. ECISs are promising alternatives to existing immunochemical tests. Their use is supported by their selectivity, sensitivity, versatility, simplicity, and short analysis time [6]. They are also renowned for their potential for miniaturization, portability, low cost, and point-of-care analysis.

The architecture and functionality of ECISs is composed of the following elements (Figure 1): working and reference electrodes, binding layer, antigen, primary antibody (analyte), labeled secondary antibody, reaction substrate and product, and measurement of current. Most ECISs developed for CD diagnosis use screen-printed gold electrodes. The use of 8-channel screen-printed electrochemical arrays simplifies the procedure and drastically improves analysis times. A huge variety of methods is available for immobilizing the target molecule (antigen) onto the electrode surface. They can be divided into covalent and noncovalent binding approaches. For example, covalent attachment can be achieved by self-assembling monolayers consisting of carboxy- or aminoterminated thiol compounds. The functional groups of the layer can be activated for covalent coupling using carbodiimide or glutaraldehyde. Noncovalent immobilization can be accomplished by polymer entrapment or by hydrophobic interactions. Another approach is based on the polyelectrolyte adsorption technique. In the last years, carbon nanostructure/metal nanoparticle hybrids have been exploited for electrochemical sensing applications. The transducer surface consists of a screen-printed carbon electrode modified with multi-walled carbon nanotubes and gold nanoparticles.

After immobilization of the antigen onto the electrode surface, the sensor is ready to use. The analytical procedure is initiated by adding the analyte (antibodies for calibration and antibodies in serum samples) onto the electrode surface. The detection of the antigen-antibody complex can be achieved with the aid of secondary antibodies. These commercially available antibodies are directed against human Igs belonging either to the IgA or to the IgG class. The secondary antibody is usually labeled with an enzyme. After adding the corresponding substrate, the enzymatic reaction product enables the quantitation of the analyte by amperometric or potentiometric measurements or by the detection of impedance changes.

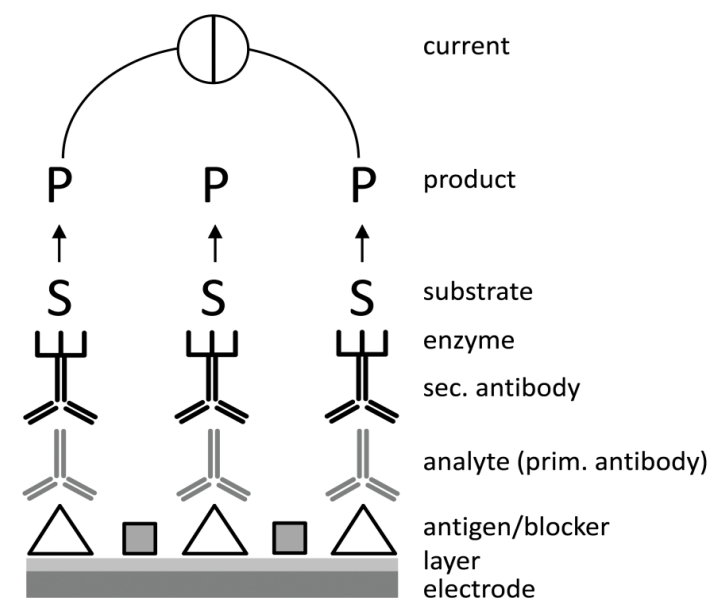

Figure 1. Schematic architecture and functionality of an electrochemical immunosensor. 


\section{Detection of Anti-Gliadin Antibodies}

The use of ECISs for the detection of AGAs was first reported by Balkenhohl and Lisdat [7]. In order to get a suitable layer for binding high amounts of gliadins to gold electrodes, different layers for the modification of the electrode surface were investigated first. The degree of immobilization was determined by quartz crystal microbalance measurements. The results demonstrated that the polyelectrolyte polystyrene sulfonic acid (PSS) was the most effective surface modification for the binding of gliadins. After the binding of gliadins onto the modified gold electrode and the blocking of unspecific and residual binding sites with bovine serum albumin (BSA), the ready-to-use electrode was applied to the detection of AGAs. The electrode was incubated with standard solutions of AGAs from rabbit serum or human serum samples followed by a washing step. The antigen-antibody interaction was amplified by incubation with horseradish peroxidase (HRP)-labeled antibodies directed against rabbit IgG and human IgG, respectively. After a final washing step, the electrode was incubated in a solution of 3-amino-9-ethylcarbazole, the substrate for HRP. The precipitating product (3-azo-9-ethylcarbazole) enhanced the insulating properties of the electrode. Subsequently, the electrode was transferred to water and the reaction product analyzed by electrochemical impedance spectrometry in the presence of ferri/ferro-cyanide using a conventional three-electrode cell. The calibration of the AGA sensor was performed using different concentrations of five human sera, which had been characterized by conventional ELISA experiments before. A sigmoidal curve showed AGA concentrations in the range of $0.008-1.5 \mu \mathrm{mol} / \mathrm{l}$.

Five different human sera, which were unambiguously characterized by ELISA - two positive for CD and three from healthy persons-were analyzed for their IgA and IgG AGA concentrations. The different sera were diluted according to the ELISA experiments in order to get values within the upper part of the calibration curve. The IgG values of the CD patients were 840 and $380 \mu \mathrm{mol} / \mathrm{l}$ and the IgA values 140 and $70 \mu \mathrm{mol} / \mathrm{l}$, respectively. The values of the healthy individuals ranged from 40 - $210 \mu \mathrm{mol} / \mathrm{l}$ (IgG) and 60 - $80 \mu \mathrm{mol} / \mathrm{l}$ (IgA). The ELISA results, given only as positive (higher than the cut-off) and negative (lower than the cut-off), were in accordance with the ECIS experiments. The only exception was the serum of a healthy individual showing a high value for IgG AGAs $(210 \mu \mathrm{mol} / \mathrm{l})$ by ECIS and a negative result by ELISA. The authors concluded that the sensor provides a reliable analytical system with an appropriate sensitivity for the analysis of human sera with respect to their AGA concentrations.

Rosales-Rivera et al. [8] introduced another ECIS system for the detection of AGAs. For binding gliadin molecules, gold disk electrodes were immersed in a solution of a carboxylic-ending bipodal alkanethiol, which presented a long polyethyleneglycol-modified chain that acted as an excellent nonspecific adsorption barrier. The bipodal nature of the thiol ensured good spacing and hence good diffusion properties through the self-assembling monolayer, which was vital for the efficiency of the constructed ECISs. The covalent binding of amino groups of gliadins and carboxyl groups of the layer was achieved by adding a mixture of N-(3-dimethylaminopropyl)-N-ethylcarbodiimide and N-hydroxysuccinimide. Unreacted remaining carboxyl groups were blocked by immersion of the electrode in an ethanolamine solution followed by a washing step. The ready-to-use electrodes were incubated with calibrator reference solutions, different AGA solutions or patients' sera followed by the incubation with corresponding HRP-labeled anti-rabbit or anti-human antibodies. The subsequent amperometric measurement was carried out in an electrochemical cell containing buffer and 3,3,5,5-tetramethylbenzidine (TMB). TMB oxidized by HRP was reduced back to its original form generating a cathodic current at the electrode.

The performance of the constructed ECIS was evaluated first with different solutions of rabbit-produced human IgG AGAs. The amperometric response showed excellent efficiency and repeatability yielding a limit of detection of $46 \mathrm{ng} / \mathrm{ml}$ with an average relative standard deviation (RSD) of 8.2\% $(\mathrm{n}=5)$. The ECIS was then compared with a commercial ELISA using reference AGA solutions from an ELISA kit and real patients' sera. The electrochemical immunosensor currents were extrapolated to the calibrator solution plot to obtain equivalent arbitrary units to those found when analyzing the same samples by commercial ELISA kits. The comparison of the results displayed a linear relationship with slope values of $>0.99$ for both IgA and IgG AGAs (Figure 2), indicative of an excellent degree of correlation. In conclusion, the ECIS matched the behavior and sensitivity achieved by a standard ELISA procedure in shorter operation time (30 min against 90 - $135 \mathrm{~min}$ ) and facilitated an excellent prospective for portability and in situ measurements.

In 2012, Neves and colleagues published the development of an ECIS based on screen-printed carbon electrodes (SCEs) modified with a carbon nanotube/gold nanoparticle hybrid system for the quantitation of IgA and 


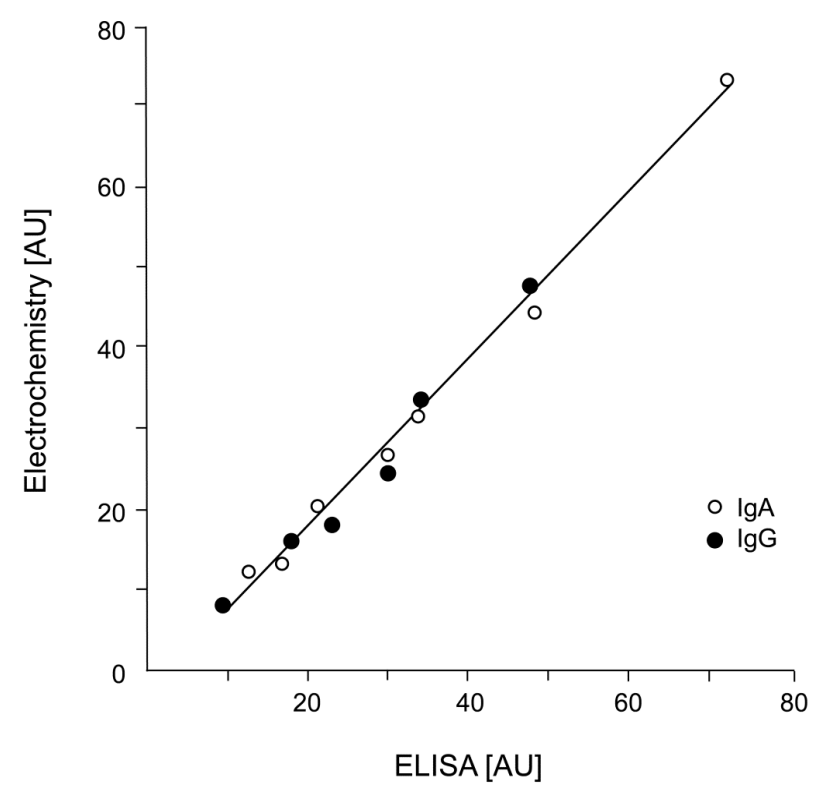

Figure 2. Comparison of the electrochemical immunosensor and commercial ELISA for the determination of anti-gliadin IgA and IgG antibodies from patients' sera (adapted from [8]) (AU = arbitrary units).

IgG AGAs in human serum [9]. Both the working and the counter electrode were made from carbon inks, screen-printed on a ceramic substrate. The nanostructuration of the working electrode was carried out using a dispersion of carboxylated multi-walled carbon nanotubes. After drying, washing and drying again, gold nanostructures were generated "in situ" over the carbon nanotube-modified electrode by applying a constant current intensity of $-5 \mu \mathrm{A}$ for $60 \mathrm{~s}$ in an acidic solution of $0.1 \mathrm{mmol} / \mathrm{l} \mathrm{AuCl}_{4}^{-}$. Finally, after washing and drying, the nanostructured electrode surface was coated by incubation with a gliadin solution and washed. Optimization studies revealed that a gliadin concentration of $1 \mathrm{mg} / \mathrm{ml}$ was the most adequate, because it presented the best compromise between the analytical signal and the background noise. Free surface sites were blocked with casein (IgA) or BSA (IgG) solutions. The dried ready-to-use sensor was incubated with serum samples, washed, incubated with anti-human IgA or anti-human IgG antibodies, labeled with alkaline phosphatase (AP) and washed. The following enzymatic reaction was carried out by dropping 3-indoxylphosphate (3-IP) and silver nitrate solutions on the sensor surface. AP hydrolyzed 3-IP resulting in an indoxyl intermediate that reduced silver ions to metallic Ag. The current, produced by the electrochemical oxidation of silver deposited on the electrode surface, was recorded using an anodic stripping cyclic voltammetry (CV) scan from $-0.002 \mathrm{~V}$ to $+0.4 \mathrm{~V}$ at a scan rate of $50 \mathrm{mV} / \mathrm{s}$.

The precision of the methodology was evaluated in terms of repeatability using a real serum sample; the results demonstrated precise results. The limits of detection (LODs) in terms of arbitrary units (AU) were 9.1 $\mathrm{AU} / \mathrm{ml}$ (IgA) and 9.0 AU/ml (IgG) and the limits of quantitation (LOQs) $30.4 \mathrm{AU} / \mathrm{ml}$ (IgA) and $31.2 \mathrm{AU} / \mathrm{ml}$ (IgG). For the evaluation of the adequacy of the immunosensors, ten different serum samples were analyzed and the results were compared with those obtained with a commercial ELISA. Samples 1 - 4 belonged to CD patients, sample 5 to a patient with selective IgA deficiency and samples 6 - 10 to healthy individuals. All the samples were qualitatively classified the same (positive or negative) by both analytical tools. The comparison of quantitative data showed some disparity, since the concentrations of IgA AGA and IgG AGA obtained by the sensor were mostly higher than those obtained with the ELISA. These differences could be due to the sample dilutions, which were performed according to the instructions for ELISA and were not optimized for the ECIS. The authors concluded that the developed ECIS is a reliable, low cost and simple methodology, being a perfect option as first platform for future transference to a point-of-care analytical device for CD diagnosis.

Initially, AGA tests developed in the 1970s and 1980s seemed very promising, but they lost diagnostic significance because their sensitivities and specificities were shown to be insufficient for the diagnosis of CD [1]. 
This disadvantage was overcome by the introduction of deamidated gliadin peptides (DGP) as antigens [10]. Therefore, an ECIS for DGPAs was developed by Neves et al. [11] based on the architecture of an ECIS for AGA detection [9] (see above). Nanohybrid SCE, secondary labeled antibodies, enzymatic reaction, and voltammetry corresponded to the conditions for AGA measurement. Four immunogenic deamidated gliadin peptides (so-called 33-mer, 26-mer, DQ2- $\gamma 1$ and DQ2- $\gamma 2$ peptides) were selected as antigens. Because these peptides, with only 33 amino acid residues as a maximum, are rather small, their immobilization onto the electrode surface was very difficult. Therefore, the antigenic peptides were covalently linked to a carrier protein [12]. Cyclic voltammograms obtained for the detection of DGPAs in a positive and a negative serum sample are shown in Figure 3. The developed ECIS was applied for the detection of IgG DGPAs in ten different serum samples (5 positive and 5 negative) and compared with the corresponding commercial ELISA. The results revealed that the ECIS presented a good qualitative correlation with the ELISA reference method for CD diagnosis.

Preliminary results on an ELISA-on-chip device, suitable for the diagnosis and follow-up of CD, were presented by Constantini et al. [13]. In this system, recognition, detection, and read-out elements were all included in a single glass substrate without external bulky and expensive equipment. The device was made of an array of amorphous silicon photosensors aligned with a pattern of poly(2-hydroxyethyl methacrylate) polymer brushes (PHEMA), which acted as anchors for the immobilization of the antigen. The PHEMA sites were treated with succinic anhydride and N-hydroxysuccinimide in the presence of 1-ethyl-3-(3-dimethylaminopropyl) carbodiimide to modify the PHEMA brushes to carboxylic and ester functions. The CD-active gliadin peptides PQPQLPYPQ and QQPQDAVQPF (one-letter-code for amino acids) were selected as antigens. A buffer solution of these peptides was poured onto the brushes and left to react for $3 \mathrm{~h}$. After washing and drying, a solution of ethanolamine was used for blocking unreacted functional groups. The PHEMA-peptide functionalized films were incubated with rabbit serum containing antibodies against the peptides. Subsequently, a solution of the secondary antibody, anti-rabbit Ig labeled with HRP, was poured onto the chip site and incubated for 15 min. The chip site was then connected to the read-out electronic device for testing the enzymatic reaction. After a drop of the chemiluminescent cocktail was spotted on the chip site, the reaction between luminol and $\mathrm{H}_{2} \mathrm{O}_{2}$ yielded a chemiluminescent signal, which was detected as photocurrent by the photosensors positioned underneath. The photocurrent signal reached a maximum at around $600 \mathrm{~s}$ and depended on the concentration of HRP of the secondary antibody. Because the ratio between the number of secondary and primary antibodies was not known, it was not possible to calculate the concentration of the primary antibodies. Therefore, photocurrent values were correlated with the HRP concentration to determine sensitivity and selectivity of the chip. The LOD was comparable with those of conventional ELISA kits. Using this approach, research will be focused on the detection of antibodies against gliadin peptides in real samples of human serum.

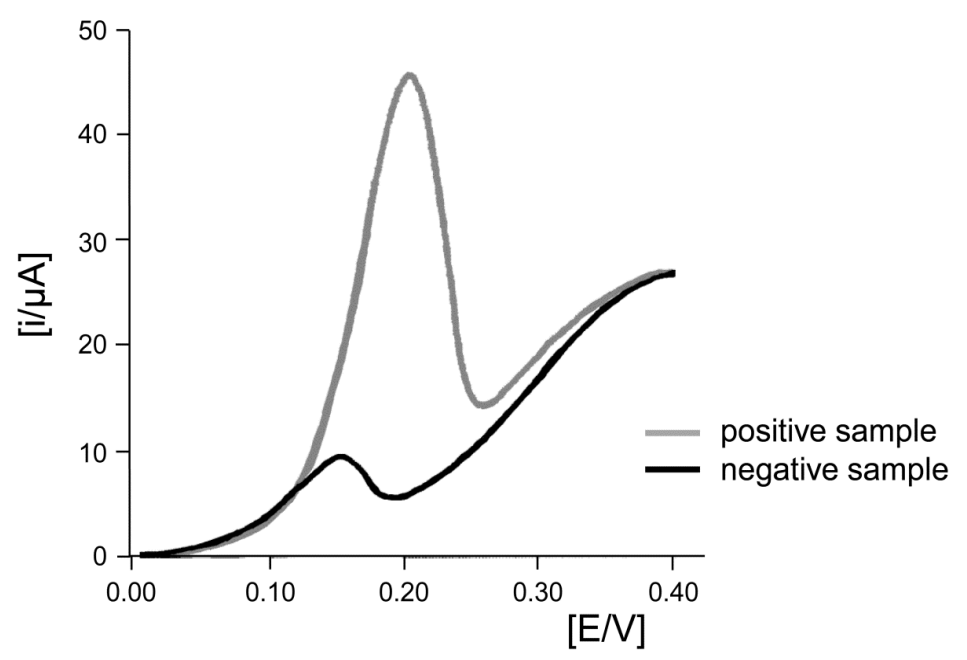

Figure 3. Cyclic voltammograms obtained for the detection of anti-deamidated gliadin peptides antibodies in a positive and a negative serum sample (adapted from [11]). 


\section{Detection of Anti-Transglutaminase Antibodies}

In 1997, TG2 was identified as the CD-specific autoantigen [14], which allowed the development of ELISAbased TGA tests, first using monkey esophagus or human umbilical cord tissue as antigen (EMA test) and then TG2 from guinea pig liver or human recombinant TG2 (TGA test) [1]. Similar to ECIS for AGA detection [7] (see Chapter 4), Balkenhohl and Lisdat developed an impedimetric immunosensor for TGA detection [15]. Screenprinted gold electrodes were layered with PSS and incubated with a solution of TG2 from guinea pig liver. Blocking, binding of serum antibodies and secondary labeled anti-IgA and anti-IgG antibodies, enzymatic reactions, and impedimetric measurements were the same as with the ECIS for AGA detection (see Chapter 4). A calibration curve was established by using different dilutions of a standard solution of TGAs from goat. Six different human sera, which had been characterized by a commercial ELISA kit (three positive and three negative), were analyzed for IgA and IgG TGAs by means of the developed ECIS. Both analytical tools showed significantly higher IgA and IgG TGA concentrations for CD patients in comparison to healthy individuals. However, quantitative differences between the values for the different sera, obtained either in the ELISA experiments or by ECIS analysis, were observed. This may have been due to less precise ECIS data in comparison to the ELISA.

Pividori and colleagues introduced an amperometric electrochemical immunosensor based on the physical adsorption of the antigen (TG2) onto a graphite-epoxy composite (GEC) electrode [16]. A three-electrode setup was used comprising a platinum auxiliary electrode, a double junction Ag/AgCl reference electrode and the working (GEC) electrode. The GEC electrode was prepared using graphite powder with a particle size of $50 \mu \mathrm{m}$, an epoxy resin and a hardener. For coating the transducer with the antigen, guinea pig liver TG2 was placed on the top of the electrode and allowed to dry. The ready-to-use sensor was incubated with serum samples to be analyzed, washed, and incubated with a secondary antibody labeled with HRP. Goat IgG fractions to human IgG, IgA, and IgM, to human IgA ( $\alpha$-chain) and to human IgG Fc (fragment crystallizable) were tested as secondary antibodies. The electrochemical signal was based on the enzymatic activity of the enzyme after the addition of $\mathrm{H}_{2} \mathrm{O}_{2}$ as the substrate and hydroquinone as the mediator. The current was measured until a steady state was reached (normally after 1 min of $\mathrm{H}_{2} \mathrm{O}_{2}$ addition) using an amperometric controller.

In total, ten positive and ten negative serum samples, controlled by a commercial ELISA method, were used to optimize analytical conditions. Different experiments were designed to evaluate the nonspecific adsorption on the immunosensor. The results demonstrated that TG2 adsorbed on GEC prevented nonspecific adsorption, so that blocking with BSA was not necessary. The best secondary antibody in terms of repeatability for the prediction of a positive serum was HRP-labeled goat IgG fraction to human IgA ( $\alpha$-chain). Among the different classes of antibodies against TG2 in human serum, the best results were achieved, when IgA TGAs were investigated. The analysis of the ten positive and ten negative serum samples revealed a sensitivity of $70 \%$ and a specificity of $100 \%$ compared with the commercial ELISA method performed in a hospital laboratory. The authors concluded that the developed ECIS offers great promise for a simple, cost-effective, and user-friendly analytical method that allows point-of-care diagnosis of CD.

Dulay et al. [17] continued the work of Rosales-Rivera et al. [8] by exchanging the antigen gliadin with the antigen TG2 for the development of an ECIS for the detection of TGAs. All other conditions (instrumentation, electrode modification, and electrochemical detection) were the same as in the former publication [8]. The immunosensor gave a stable quantitative response to different antibody concentrations after 30 min with a LOD of $390 \mathrm{ng} / \mathrm{ml}$ and an RSD of $9 \%(n=3)$. The ECIS was tested with calibrator solutions as well as with real patient samples. The results showed an excellent degree of correlation compared to those obtained from commercial IgA and IgG ELISA kits. The sensor clearly demonstrated the great potential of the dithiol-based surface chemistry modification to produce a rapid, reliable and low cost platform for the detection of antibodies related to CD.

Overoxidized polypyrrole (OOPP) doped with gold nanoparticles (GNP) on a glassy carbon electrode was used by West et al. [18] as a platform to construct a novel label-free impedimetric immunosensor for TGA determination. Field emission scanning electron microscopy results confirmed the deposition of GNP with good surface coverage across the OOPP substrate. The average diameter of the nanocomposite was $100 \mathrm{~nm}$. The platform was conductive and exhibited reversible electrochemistry. The electrochemical characterization of the platform was studied by CV and square wave voltammetry (SWV). The SWV results corroborated CV findings, which showed enhanced peak current through GNP. The immunosensor was prepared by immobilizing $40 \mu \mathrm{l}$ TG2 $(0.3 \mathrm{mg} / \mathrm{ml})$ onto the platform by drop coating. Electrochemical impedance spectroscopy measurements indicated that the immunosensor system markedly improved the conductivity and response of the TGA 
immunosensor. The impedimetric immunosensor exhibited a charge transfer resistance-dependent dynamic linearity of $10^{-6}$ to $10^{-4} \mathrm{~mol} / \mathrm{l}$ for the target TGA $(\mathrm{r}=0.9808)$ and a LOD of $5.22 \times 10^{-6} \mathrm{~mol} / \mathrm{l}$.

The practical application of the immunosensor was demonstrated by establishing a calibration curve with different dilutions (1:200 - 1:4000) of a TGA standard solution. The correlation value between the impedimetric signals and the TGA concentration was found to be 0.98 . The authors concluded that the developed ECIS holds great promise for the future as an alternative to ELISA.

Corresponding to the development of an ECIS for the detection of AGAs [9] (see above), Neves et al. [19] used SCEs, nanostructurized with nanotubes and gold nanoparticles, for the detection of TGAs. Again, the immunosensing strategy consisted of the immobilization of the antigen (TG2) on the nanostructured electrode surface followed by the electrochemical detection of TGAs present in serum samples using AP-labeled anti-human IgA or IgG antibodies. The enzymatic reaction was carried out by incubation of the sensor surface with a solution of 3-IP and silver ions. The metallic silver deposited on the electrode was detected by CV.

A precision study using positive as well as negative serum samples indicated good repeatability, with an RSD of $9.3 \%$ for the negative samples and $2.0 \%$ for the positive samples. The comparison of the developed immunosensor with a commercial ELISA, using four positive and six negative sera, did not perfectly agree, however, the qualitative results (i.e., positive or negative) matched perfectly. Thus, the authors proposed that the developed immunosensor is a trustful analytical screening tool.

A magneto-electrochemical immunosensor was developed by Adornetto et al. [20]. The portable instrumentation consisted of a sensor array with eight SCEs supported by eight neodymium magnets on the working electrode surfaces. TG2-coated magnetic beads served as support for the immunological chain including the antigen, the primary antibody (the analyte), and the secondary antibody (anti-human IgA labeled with AP). The modified magnetic beads were confined on the surfaces of the working electrode with the aid of the magnets. Then, the enzyme substrate, 1-naphthyl phosphate, was added and left to react for 2 min. The enzymatic product, 1-naphthol, was then measured by differential pulse voltammetry.

After optimizing the experimental conditions, calibration curves with four different levels of IgA TGAs (4, 10, 25, $50 \mathrm{AU} / \mathrm{ml}$ ) were used to assess precision and recovery of the proposed method (3 replicates, 3 days, 2 electrodes). The recoveries were $96 \%-100 \%$ and the coefficient of variation $13 \%-25 \%$. The LOD was found to be $0.7 \mathrm{AU} / \mathrm{ml}$ and the LOQ was $1.0 \mathrm{AU} / \mathrm{ml}$. Finally, in order to assess the agreement between the sensor and a conventional ELISA kit, a correlation curve was calculated using 26 human serum samples measured by both methods. $50 \mathrm{AU} / \mathrm{ml}$ was the upper limit of linearity for both methods. The determined coefficient of regression was $r^{2}=0.943$. Compared with ELISA, the developed sensor was characterized by similar specificity and sensitivity, a lower cut-off and a shorter analysis time (30 vs. $120 \mathrm{~min}$ ). According to the authors' conclusion, the sensor is less expensive, smaller, and suitable for analysis performed outside the classic diagnostic laboratory.

A second electrochemical magneto immunosensor for the detection of TGAs was presented by Kergaravat $e t$ al. [21]. Two electrochemical cells were tested. The first cell was constituted by a system of three independent electrodes: a graphite-epoxy composite cylindrical electrode (c-GEC) with a magnetic connection as working electrode; a platinum wire as auxiliary electrode and a $\mathrm{Ag} / \mathrm{AgCl}$ electrode as reference electrode. The second cell was constituted by disposable screen-printed electrodes formed by graphite electrodes as working electrode and counter electrodes, a silver electrode as reference electrode and a magnetic connector. Taking the advantages into consideration, the c-GEC system was chosen as transducer in the immunosensor. The immunological reaction was performed on magnetic beads as a solid support, on which the antigen (TG2 from guinea pig liver) was covalently immobilized. TGAs (whole antiserum produced in goat) were used as a model analyte. Anti-goat IgG, labeled with HRP, served as secondary antibody. The TG2-coated magnetic beads were incubated with TGAs and after washing, the anti-goat IgG labeled with HRP was added. After a second washing step, the beads were resuspended and captured by dipping the c-GEC electrodes into the suspension. The c-GEC electrode was put in the reaction cell with the enzymatic cosubstrates o-phenylendiamine and $\mathrm{H}_{2} \mathrm{O}_{2}$. The electrochemical response was obtained by SWV in the range of 0 to $-400 \mathrm{mV}$.

Assay validation performed with whole TG2 anti-serum resulted in intra-assay and interassay precision given as RSD from $10 \%$ to $13 \%$ and from $14 \%$ to $16 \%$, respectively. Regarding accuracy and repeatability, recoveries ranged from $94 \%$ to $123 \%$. For the analysis of human serum samples, the anti-goat secondary antibody was exchanged by an anti-human IgA antibody. A number of 29 sera from clinically confirmed CD cases and 19 negative control sera were tested by the developed sensor. The data indicated that $16.95 \mathrm{AU} / \mathrm{ml}$ was the most effective cut-off value to discriminate correctly between celiac and nonceliac individuals. Using this point for predic- 
tion, sensitivity was found to be $100 \%$, while specificity was $84 \%$. According to the authors' plans for future developments, the assay should improve its specificity, e.g., by exchanging the antigen with human recombinant TG2, and by including nanoparticles into the transducer.

A miniaturized ECIS with quantum dots (QDs) was developed for the detection of IgG TGAs in human sera by Martin-Yerga et al. [22]. QDs are semiconductor nanoparticles and the metallic component can be measured electrochemically after an acid attack to break the nanoparticle and release metal cations into the solution. For the production of the sensor, an 8-channel screen-printed electrochemical array was formed by eight 3-electrode cells with carbon-based working and counter electrodes. In the present work, CdSe/ZnS QDs were used and $\mathrm{Cd}^{2+}$ release was measured by differential pulse voltammetry. For antigen binding, a TG2 solution was dropped on the surface of each working electrode, and after washing, a blocking step was carried out by placing a casein solution on the surface. The ready-to-use sensor was incubated with human serum samples followed by a washing step. Finally, goat anti-human-IgG, labeled with QDs, was dropped on the electrodes and washed. Then, the electrode was connected to the potentiostat, and $\mathrm{HCl}$ was added on the working electrode followed by the addition of a $\mathrm{Bi}(\mathrm{III})$ solution. A constant potential of $+1.00 \mathrm{~V}$ was applied for $60 \mathrm{~s}$ to activate the electrode. Cadmium was preconcentrated on the electrode surface by applying a potential of $-1.10 \mathrm{~V}$ for $300 \mathrm{~s}$; simultaneously, a bismut film was also formed during this step. The potential was swept from $-1.10 \mathrm{~V}$ to $-0.65 \mathrm{~V}$ using differential pulse voltammetry.

After optimization of the experimental conditions (e.g., concentrations of antigen, blocking reagent, serum samples, and antibodies as well as reaction times), the developed sensor was used to establish a relationship between the analytical signal and the concentration of IgG TGAs using human serum calibrators from a commercial ELISA kit (0, 7, 16 and $40 \mathrm{AU} / \mathrm{ml})$ (Figure 4). The detection limit of the sensor was $2.2 \mathrm{AU} / \mathrm{ml}$ and the RSD of the slopes of calibration curves was 5.9\%. The stability of the sensors was tested over a 1-month period, the results obtained confirmed that they were stable for at least one month. According to the authors' conclusion, the developed ECIS is a trustful screening tool for CD diagnosis, which allows discrimination between positive and negative serum samples with high sensitivity.

Antibodies against the open (active) conformation of TG2 were shown to be more specific than antibodies against the closed form, especially in modest cases of CD and limited gluten exposure and for the evaluation of the adherence to a GFD [23]. Therefore, an amperometric immunosensor, based on the covalent immobilization of TG2 in its open conformation, was developed for the determination of TGAs in human sera by Giannetto et al. [24]. Sensors were assembled on a glassy carbon disc electrode. A platinum rod electrode and $\mathrm{Ag} / \mathrm{AgCl} / \mathrm{KCl}$ were used as counter and reference electrodes, respectively. The carbon electrode was electrochemically functionalized with gold nanoparticles and subsequently derivatized with a self-assembling monolayer of 11-mercaptoundecanoic acid for the covalent anchoring of open TG2, which was conformationally stabilized through covalent binding of 6-diazo-5-oxo-L-norleucine. The covalent linking of TG2 was performed by combining the use of a carbodiimide compound and N-hydroxy-succinimide. Afterwards, blocking of remaining active sites was performed with BSA. The ready-to-use-electrode was dried and stored at $4^{\circ} \mathrm{C}$. Detection of TGAs was carried out by incubating the sensor with diluted serum samples, followed by incubation with anti-human IgA or IgG labeled with HRP. After addition of thionin and $\mathrm{H}_{2} \mathrm{O}_{2}$, CV reading was performed by scanning the potential

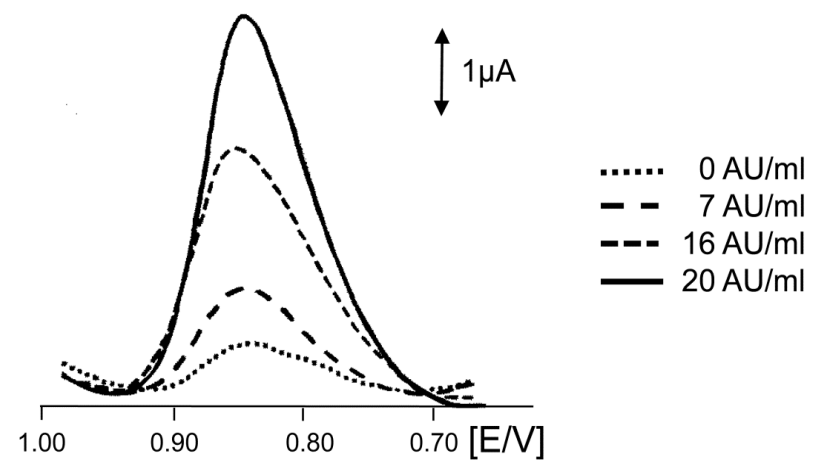

Figure 4. Differential-pulse voltammograms for different calibrators according to anti-TG2 IgG antibody concentration (adapted from [22]). 
between -0.2 and $+0.6 \mathrm{~V}$ at a scan rate of $0.05 \mathrm{~V} / \mathrm{s}$.

Using the calibration solutions $(0,1,3,10,30,100 \mathrm{AU} / \mathrm{ml})$ of a commercial ELISA kit, the sensor showed good analytical performance with LODs (1.7 AU/ml for IgA and $2.7 \mathrm{AU} / \mathrm{ml}$ for IgG) below the diagnostic threshold value $(3.0 \mathrm{AU} / \mathrm{ml})$. The linearity of the response was assessed over 0 - $30 \mathrm{AU} / \mathrm{ml}$. Intersensor repeatability was lower than $10 \%$. The sensor was further validated by the analysis of serum samples from ten pediatric patients using two commercial ELISA kits specific for the determination of IgA and IgG TGAs (Table 1). Very good recovery rate values, referred to data from the commercial ELISA kits, were achieved within the clinically useful concentration range. This confirmed the clinical applicability of the developed immunoassay to patients' serum samples.

\section{Simultaneous Detection of Anti-Gliadin and Anti-Transglutaminase Antibodies}

Several studies on the performance and diagnostic accuracy of commercial ELISA kits demonstrated that the clinical demand for both maximal sensitivity and maximal specificity cannot be achieved by a single test. The combination of different tests, e.g., Ig TGA and IgG DGPA should be adapted to screen for CD [25]. Therefore, a dual ECIS for the simultaneous detection of AGAs and TGAs in patient samples was developed by Neves et al. [26]. The sensor was based on two ellipse-shaped carbon working electrodes nanostructured with a carbon-gold hybrid system, a carbon counter electrode and a silver pseudo-reference electrode, all of them screen-printed on a ceramic substrate. The immunosensing strategy comprised all steps included in previous developments of sensors for separate AGA and TGA determinations [9] [19]: immobilization of the antigens (gliadin and TG2) onto the working electrode surfaces, blocking of remaining active sites by BSA, addition of the primary antibodies (calibrators or diluted sera), addition of the secondary antibodies (anti-human Ig labeled with AP), addition of the enzymatic substrate (3-IP) and silver ions, and measurement by CV.

After optimizing the system, the precision of the methodology was evaluated in terms of repeatability, which was assessed through three inter-day evaluations. In case of IgA detection, RSD values of $7.5 \%$ and $3.7 \%$ were obtained for AGA and TGA detection, respectively. For IgG detection, RSD values of 7.2\% (AGA) and 4.3\% (TGA) were obtained. Using human sera calibrators, a linearity between current intensity and the immunoglobulin content (in arbitrary units) was achieved between 0 and $100 \mathrm{AU} / \mathrm{ml}$. In case of IgA TGA detection, the LOD

Table 1. Determination of TGAs in human serum samples and positive/negative controls by ECIS and ELISA ${ }^{\mathrm{a}}$.

\begin{tabular}{ccccc}
\hline \multirow{2}{*}{ Sample } & \multicolumn{2}{c}{ IgA TGA $[\mathrm{AU} / \mathrm{ml}]$} & \multicolumn{2}{c}{ IgG TGA [AU/ml] } \\
\cline { 2 - 5 } & ECIS & ELISA & ECIS & ELISA \\
\hline S1 & $6.5 \pm 0.5$ & $8.8 \pm 0.3$ & n.d. & n.d. \\
S2 & $29.0 \pm 0.8$ & $34.2 \pm 0.2$ & $20.1 \pm 0.7$ & $21.9 \pm 0.3$ \\
S3 & n.d. & n.d. & $122.9 \pm 2.1$ & $116.0 \pm 5.4$ \\
S4 & n.d. & n.d. & $627.5 \pm 3.3$ & $653.7 \pm 4.2$ \\
S5 & $8.4 \pm 0.6$ & $10.8 \pm 0.6$ & n.d. & n.d. \\
S6 & $8.7 \pm 0.5$ & $10.7 \pm 1.0$ & n.d. & n.d. \\
S7 & $35.5 \pm 0.9$ & $33.2 \pm 0.6$ & n.d. & n.d. \\
S8 & n.d. & n.d. & 7.6 \pm 0.8 & $7.8 \pm 0.3$ \\
S9 & n.d. & n.d. & n.d. & n.d. \\
S10 & n.d. & n.d. & n.d. & n.d. \\
Pos.control & 26.7 \pm 0.1 & $22.7 \pm 0.2$ & $27.7 \pm 0.6$ & 26.7 \pm 0.1 \\
Neg.control & n.d. & n.d. & n.d. & n.d. \\
\hline
\end{tabular}

${ }^{\mathrm{a}}$ Adapted from Giannetto et al. [24]; ${ }^{\mathrm{b}}$ Not detected (below limit of detection). 


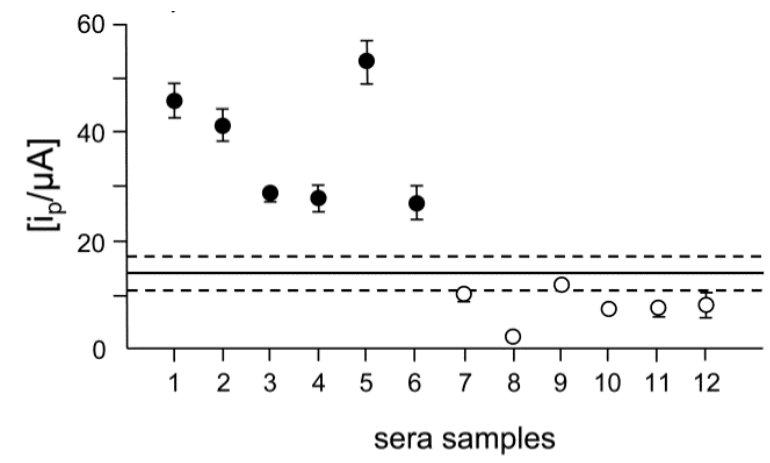

(a)

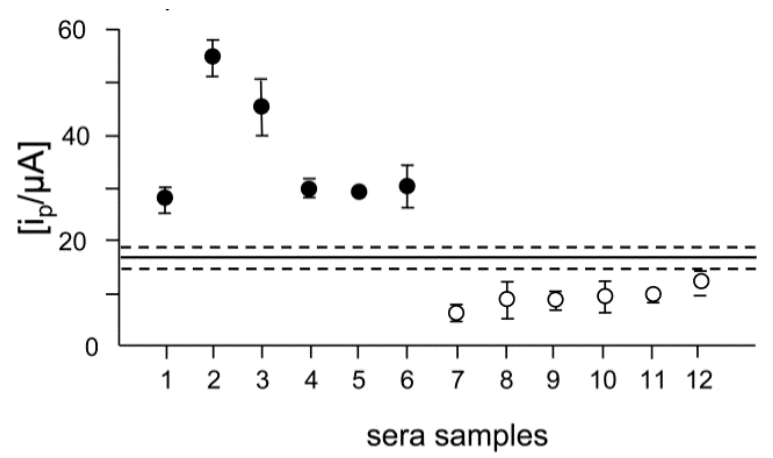

(b)

Figure 5. Evaluation of six positive (black filled circles) and six negative (unfilled circles) human serum samples for the simultaneous detection of anti-TG2 IgA (a) and anti-gliadin IgA (b) antibodies (adapted from [26]).

and LOQ values obtained were $2.45 \mathrm{AU} / \mathrm{ml}$ and $20.42 \mathrm{AU} / \mathrm{ml}$, respectively. In case of IgG TGA detection, LOD and LOQ were found to be $2.95 \mathrm{AU} / \mathrm{ml}$ and $37.15 \mathrm{AU} / \mathrm{ml}$, respectively. LOD and LOQ values were $3.16 \mathrm{AU} / \mathrm{ml}$ and $46.77 \mathrm{AU} / \mathrm{ml}$ for IgA AGA detection and $2.82 \mathrm{AU} / \mathrm{ml}$ and $32.36 \mathrm{AU} / \mathrm{ml}$ for IgG AGA determination. In order to establish an agreement between the dual sensor and a commercial ELISA, twelve different serum samples were analyzed with both methods. Figure 5 shows the obtained data for the detection of IgA TGAs and IgA AGAs. Altogether, the results revealed that the dual sensor gave some slightly higher values than ELISA. Nevertheless, the comparison of the results indicated an excellent degree of correlation between both methodologies. The authors concluded that the developed sensor can be a good alternative to the traditional methods allowing a decentralization of the analyses towards a point-of-care strategy.

\section{Conclusion}

In the last years, a remarkable progress in the development of ECISs for the diagnosis of CD has been made. Recent results on specificity and sensitivity suggest that in the future, ECISs can be promising alternatives to existing immunochemical tests, e.g., traditional ELISAs. They are simple, reliable, robust, user-friendly, and cost-effective analytical tools with short operation times and can be a perfect option as the first platform for transference to a point-of-care analytical device for CD diagnosis. However, some questions remain, such as sufficient accuracy, repeatability, reproducibility and long-term stability. Performance criteria for specificity, sensitivity, handling, time and cost of analysis should be established for each single system and compared to traditional ELISA methods. Finally, international collaborative studies should clarify which of the developed ECISs is the best candidate for substituting ELISA.

\section{References}

[1] Wieser, H., Koehler, P. and Konitzer, K. (2014) Celiac Disease and Gluten. Multidisciplinary Challenges and Opportunities. Academic Press, Amsterdam, New York, San Diego.

[2] Husby, S., Koletzko, S., Korponay-Szabo, I.R., Mearin, M.L., Phillips, A., Shamir, R., Troncone, R., Giersiepen, K., Branski, D., Catassi, C., Lelgeman, M., Mäki, M., Ribes-Koninckx, C., Ventura, A. and Zimmer, K.P. (2012) European Society for Pediatric Gastroenterology, Hepatology, and Nutrition Guidelines for the Diagnosis of Coeliac Disease. Journal of Pediatric Gastroenterology and Nutrition, 54, 136-160. http://dx.doi.org/10.1097/MPG.0b013e31821a23d0

[3] Giersiepen, K., Lelgemann, M., Stuhldreher, N., Ronfani, L., Husby, S., Koletzko, S. and Korponay-Szabo, I.R. (2012) Accuracy of Diagnostic Antibody Tests for Coeliac Disease in Children: Summary of Evidence Report. Journal of Pediatric Gastroenterology and Nutrition, 54, 229-241. http://dx.doi.org/10.1097/MPG.0b013e318216f2e5

[4] Rozenberg, O., Lerner, A., Pacht, A., Grinberg, M., Reginashvilli, D., Hening, C. and Barak, M. (2012) A Novel Algorithm for the Diagnosis of Celiac Disease and a Comprehensive Review of Celiac Disease Diagnostics. Clinical Reviews in Allergy \& Immunology, 42, 331-341. http://dx.doi.org/10.1007/s12016-010-8250-y

[5] Luppa, P.B., Sokoll, L.J. and Chan, D.W. (2001) Immunosensors-Principles and Applications to Clinical Chemistry. Clinica Chimica Acta, 314, 1-26. http://dx.doi.org/10.1016/S0009-8981(01)00629-5

[6] Bange, A., Halsall, H.B. and Heineman, W.R. (2005) Microfluidic Immunosensor Systems. Biosensors and Bioelectronics, 20, 2488-2503. http://dx.doi.org/10.1016/j.bios.2004.10.016 
[7] Balkenhohl, T. and Lisdat, F. (2007) An Impedimetric Immunosensor for the Detection of Autoantibodies Directed against Gliadins. Analyst, 132, 314-322. http://dx.doi.org/10.1039/b609832k

[8] Rosales-Rivera, L.C., Acero-Sanchez, J.L., Lozano-Sanchez, P., Katakis, I. and O’Sullivan, C.K. (2011) Electrochemical Immunosensor Detection of Antigliadin Antibodies from Real Human Sera. Biosensors and Bioelectronics, 26, 4471-4476. http://dx.doi.org/10.1016/j.bios.2011.05.004

[9] Neves, M.M.P.S., Gonzales-Garcia, M.B., Santos-Silva, A. and Costa-Garcia, A. (2012) Voltammetric Immunosensors for the Diagnosis of Celiac Disease Based on the Quantification of Anti-Gliadin Antibodies. Sensors and Actuators B, 163, 253-259. http://dx.doi.org/10.1016/j.snb.2012.01.048

[10] Schwertz, E., Kahlenberg, F., Sack, U., Richter, T., Stern, M., Conrad, K., Zimmer, K.-P. and Mothes, T. (2004) Serologic Assay Based on Gliadin-Related Nonapeptides as a Highly Sensitive and Specific Diagnostic Aid in Celiac Disease. Clinical Chemistry, 50, 2370-2375. http://dx.doi.org/10.1373/clinchem.2004.036111

[11] Neves, M.M.P.S., Gonzales-Garcia, M.B., Nouws, H.P.A. and Costa-Garcia, A. (2013) An Electrochemical Deamidated Gliadin Antibody Immunosensor for Celiac Disease Clinical Diagnosis. Analyst, 138, 1956-1958. http://dx.doi.org/10.1039/c3an36728b

[12] Dorum, S., Qiao, S.W., Sollid, L.M. and Fleckenstein, B. (2009) A Quantitative Analysis of Transglutaminase 2-Mediated Deamidation of Gluten Peptides: Implications for the T-Cell Response in Celiac Disease. Journal of Proteome Research, 8, 1748-1755. http://dx.doi.org/10.1021/pr800960n

[13] Constantini, F., Nascetti, A., Scipinotti, R., Domenici, F., Sennato, S., Gazza, L., Bordi, F., Pogna, N., Manetti, C., Caputo, D. and De Cesare, G. (2014) On-Chip Detection of Multiple Serum Antibodies against Epitopes of Celiac Disease by an Array of Amorphous Silicon Sensors. RCS Advances, 4, 2073-2080.

[14] Dieterich, W., Ehnis, T., Bauer, M., Donner, P., Volta, U., Riecken, E.O. and Schuppan, D. (1997) Identification of Tissue Transglutaminase as the Autoantigen of Celiac Disease. Nature Medicine, 3, 797-801. http://dx.doi.org/10.1038/nm0797-797

[15] Balkenhohl, T. and Lisdat, F. (2007) Screen-Printed Electrodes as Impedimetric Immunosensors for the Detection of Anti-Transglutaminase Antibodies in Human Sera. Analytica Chimica Acta, 597, 50-57. http://dx.doi.org/10.1016/j.aca.2007.06.041

[16] Pividori, M.I., Lermo, A., Bonnani, A., Alegret, S. and Del Valle, M. (2009) Electrochemical Immunosensor for the Diagnosis of Celiac Disease. Analytical Biochemistry, 388, 229-234. http://dx.doi.org/10.1016/j.ab.2009.02.026

[17] Dulay, S., Lozano-Sanchez, P., Iwuoha, E., Katakis, I. and O’Sullivan, C.K.O. (2011) Electrochemical Detection of Celiac Disease-Related Anti-Tissue Transglutaminase Antibodies Using Thiol Based Surface Chemistry. Biosensors and Bioelectronics, 26, 3852-3856. http://dx.doi.org/10.1016/j.bios.2011.02.045

[18] West, N., Baker, P.G.L., Arotiba, O.A., Hendricks, N.R., Baleg, A.A., Waryo, T.T., Ngece, R.F., Iwuoha, E.I. and O’Sullivan, C. (2011) Overoxidized Polypyrrole Incorporated with Gold Nanoparticles as Platform for Impedimetric Anti-Transglutaminase Immunosensor. Analytical Letters, 44, 1956-1966. http://dx.doi.org/10.1080/00032719.2010.539739

[19] Neves, M.M.P.S., Gonzales-Garcia, M.B., Nouws, H.P.A. and Costa-Garcia, A. (2012) Celiac Disease Detection Using a Transglutaminase Electrochemical Immunosensor Fabricated on Nanohybrid Screen-Printed Carbon Electrodes. Biosensors and Bioelectronics, 31, 95-100. http://dx.doi.org/10.1016/j.bios.2011.09.044

[20] Adornetto, G., Volpe, G., De Stefano, A., Martini, S., Gallucci, G., Manzoni, A., Bernardini, S., Mascini, M. and Moscone, D. (2012) An ELIME Assay for the Rapid Diagnosis of Coeliac Disease. Analytical and Bioanalytical Chemistry, 403, 1191-1194. http://dx.doi.org/10.1007/s00216-011-5702-z

[21] Kergaravat, S.V., Beltramino, L., Garnero, N., Trotta, L., Wagener, M., Pividori, M.I. and Hernandez, S.R. (2013) Electrochemical Magneto Immunosensor for the Detection of Anti-TG2 Antibody in Celiac Disease. Biosensors and Bioelectronics, 48, 203-209. http://dx.doi.org/10.1016/j.bios.2013.04.012

[22] Martin-Yerga, D., Gonzales-Garcia, M.B. and Costa-Garcia, A. (2014) Electrochemical Immunosensor for Anti-Tissue Transglutaminase Antibodies Based on the in Situ Detection of Quantum Dots. Talanta, 130, 598-602. http://dx.doi.org/10.1016/j.talanta.2014.07.010

[23] Pallav, K., Leffler, D.A., Bennett, M., Tariq, S., Xu, H., Kabbani, T., Moss, A.C., Dennis, M., Kelly, C.P. and Schuppan, D. (2012) Open Conformation Tissue Transglutaminase Testing for Celiac Dietary Assessment. Digestive and Liver Disease, 44, 375-378. http://dx.doi.org/10.1016/j.dld.2011.12.008

[24] Giannetto, M., Mattarozzi, M., Umilta, E., Manfredi, A., Quaglia, S. and Careri, M. (2014) An Amperometric Immunosensor for Diagnosis of Celiac Disease Based on Covalent Immobilization of Open Conformation Tissue Transglutaminase for Determination of Anti-tTG Antibodies in Human Serum. Biosensors and Bioelectronics, 62, 325-330. http://dx.doi.org/10.1016/j.bios.2014.07.006

[25] Bizzaro, N., Tozzoli, R., Villalta, D., Fabris, M. and Tonutti, E. (2012) Cutting-Edge Issues in Coeliac Disease and in 
Gluten Intolerance. Clinical Reviews in Allergy \& Immunology, 42, 279-287. http://dx.doi.org/10.1007/s12016-010-8223-1

[26] Neves, M.M.P.S., Gonzales-Garcia, M.B., Delerue-Matos, C. and Costa-Garcia, A. (2013) Multiplexed Electrochemical Immunosensor for Detection of Celiac Disease Serological Markers. Sensors and Actuators B, 187, 33-39.

http://dx.doi.org/10.1016/j.snb.2012.09.019 
Scientific Research Publishing (SCIRP) is one of the largest Open Access journal publishers. It is currently publishing more than 200 open access, online, peer-reviewed journals covering a wide range of academic disciplines. SCIRP serves the worldwide academic communities and contributes to the progress and application of science with its publication.

Other selected journals from SCIRP are listed as below. Submit your manuscript to us via either submit@scirp.org or Online Submission Portal.
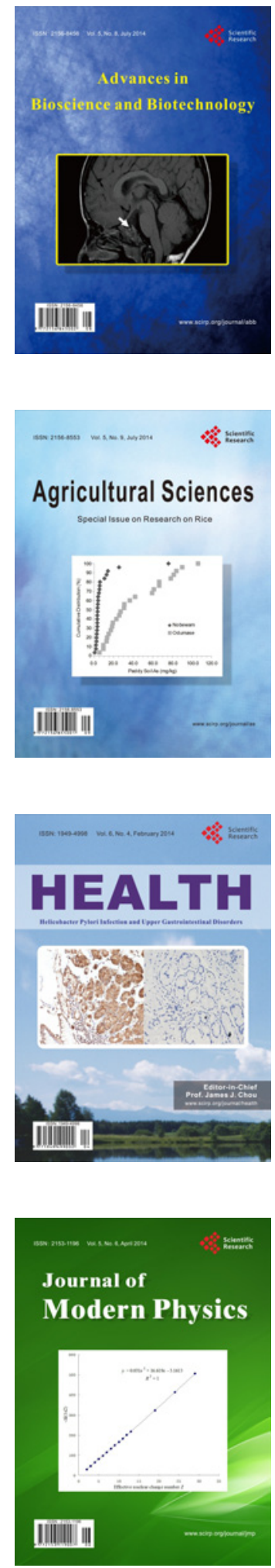
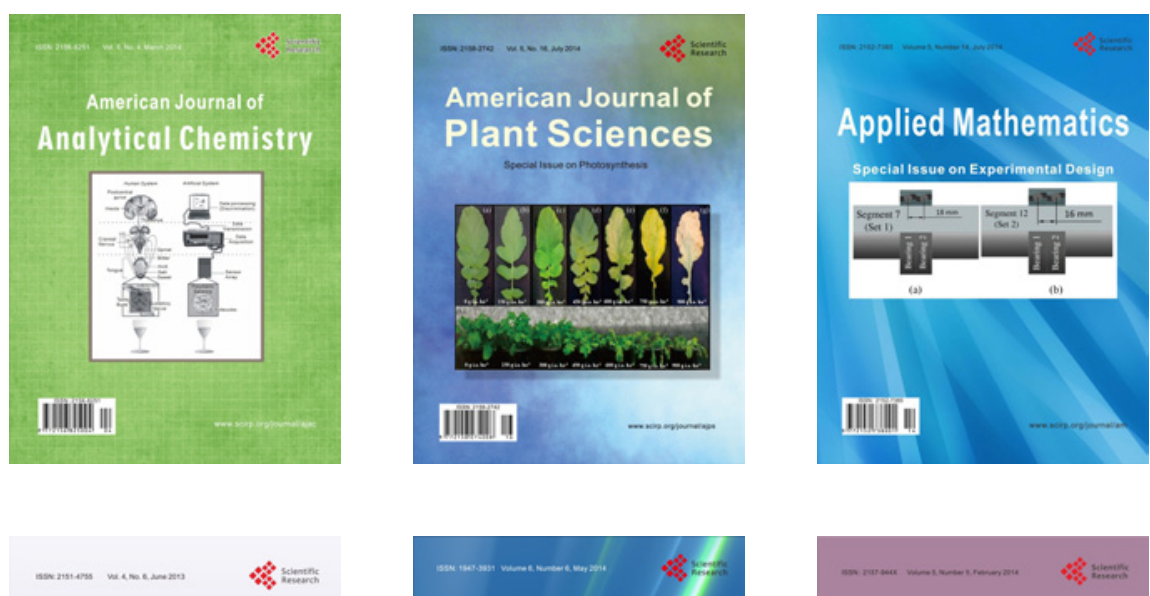

Creative Education
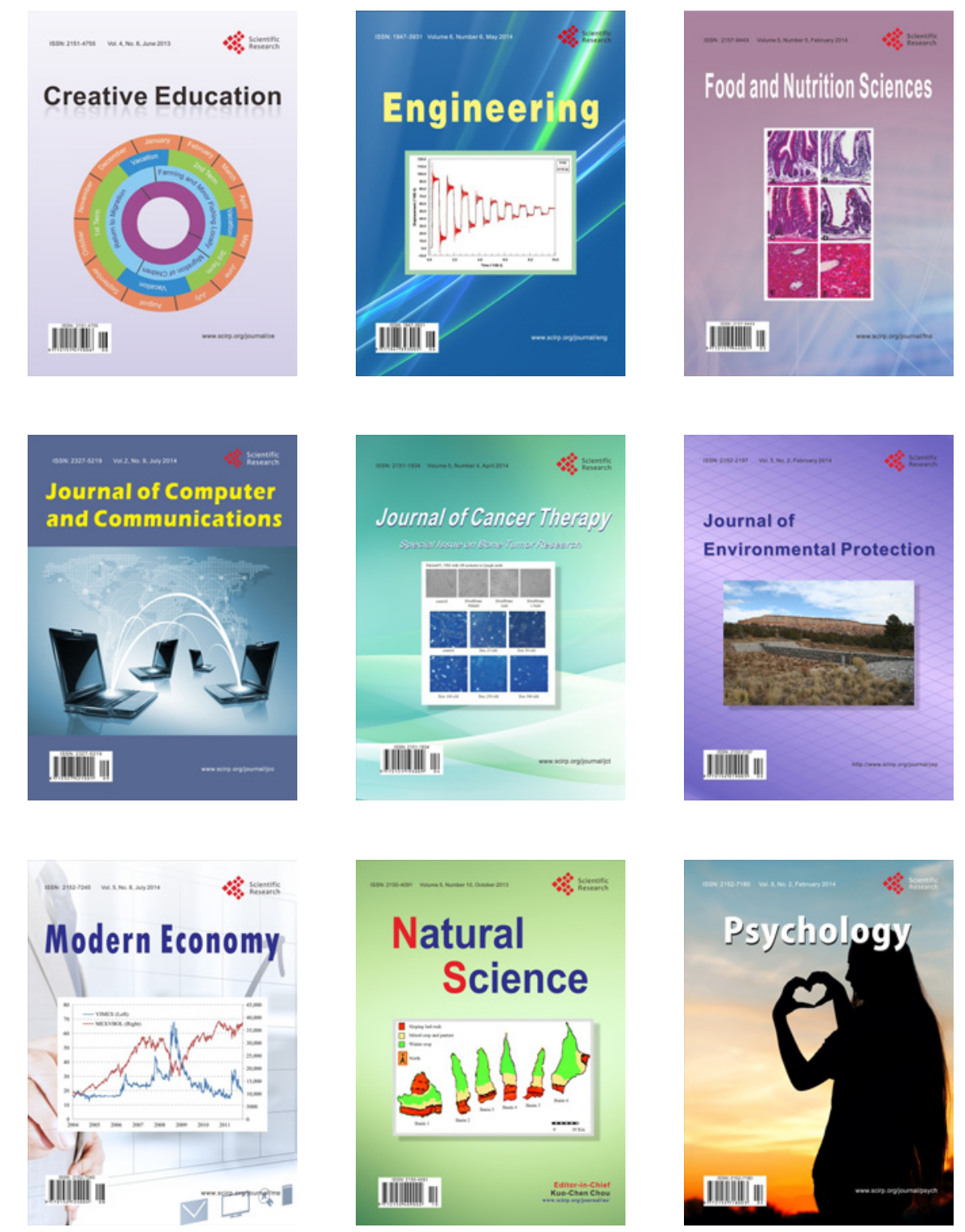